\title{
Receiver Configuration and Testbed Development for Underwater Cognitive Channelization
}

\author{
George Sklivanitis, ${ }^{*}$ Emrecan Demirors, ${ }^{\dagger}$ Stella N. Batalama, ${ }^{*}$ Tommaso Melodia, ${ }^{\dagger}$ and Dimitris A. Pados* \\ ${ }^{*}$ Department of Electrical Engineering, The State University of New York at Buffalo, Buffalo, NY 14260-2050. \\ E-mail:\{gsklivan, batalama, pados\}@buffalo.edu \\ ${ }^{\dagger}$ Department of Electrical and Computer Engineering, Northeastern University, Boston, MA 02115. \\ E-mail:\{edemirors, melodia\}@ece.neu.edu
}

\begin{abstract}
We propose a receiver configuration and we develop a software-defined-radio testbed for real-time cognitive underwater multiple-access communications. The proposed receiver is fully reconfigurable and executes (i) all-spectrum cognitive channelization and (ii) combined synchronization, channel estimation, and demodulation. Online (real-time) experimental field studies using in-house built modems demonstrate our theoretical developments and show that cognitive channelization is a powerful proposition for underwater communications that leads to significant improvement of spectrum utilization. Even in the absence of interference, due to the noise characteristics of the acoustic channel, cognitive channelization offers significant performance improvements in terms of receiver pre-detection signal-to-interference-plus-noise-ratio and bit-error-rate.
\end{abstract}

\section{INTRODUCTION}

Underwater Acoustic (UW-A) communications has been an emerging research topic for the last few years due to its wide range of commercial and military applications such as tactical surveillance, marine life monitoring, and offshore exploration. The UW-A channel poses several challenges such as high path loss, noise, severe multipath, Doppler spread, high and variable propagation delay, and limited bandwidth that is both temporally and spatially varying [1].

As of today, the limited bandwidth of UW-A channel is heavily utilized by multiple "artificial" acoustic systems (i.e., sonar users) for a variety of underwater applications (e.g., echolocation, sensing) as well as shared with "natural" acoustic systems such as marine mammals. However, spectrum resources remain underutilized both spatially and temporally [2]. At the same time, existing underwater modems lack the ability for channel-awareness and are not sufficiently flexible to dynamically reconfigure their communication parameters in real-time (e.g., modulation scheme, frequency band, and waveform). Therefore, cognitive acoustics need to overcome both practical challenges and the unique features that characterize the rapidly-varying underwater environment.

In contrast to terrestrial radio networks where cognitive radio is a well-researched topic [3], [4], there are only few works that consider cognitive underwater acoustic networks [5]-[7]. In particular, work in [5] borrows different dynamic spectrum access models from terrestrial networks and investigates their potential application to heterogeneous underwater network setups. In [6] authors assume known topology and distance information of underwater nodes, and provide a frequency allocation algorithm to maximize fairness and efficiency of spectrum utilization. Finally, the work in [7] extends the treatment of [6] for unknown topology but still known distance information.

This work was supported in part by the National Science Foundation under grants CNS-1422874 and CNS-1126357.
In this work, we study a new paradigm for underwater spectrum access, based on all-spectrum cognitive channelization [3], and we propose and implement a complete receiver configuration that executes cognitive channelization and combined synchronization, channel estimation, and demodulation in a blind/unsupervised fashion. Our theoretical developments are evaluated in a custom software-defined radio (SDR) underwater testbed. In particular, our testbed consists of three in-house built software-defined acoustic modems that are based on the Universal Software Radio Peripheral (USRP-N210) platform, and are controlled by the GNU Radio software framework on host-PCs. Experiments take place in the underwater environment of a test tank.

More specifically, we consider a communication link coexisting with multiple underwater ("artificial" or "natural") signals. Channel access is achieved through real-time selection of optimized waveforms that maximize the output signalto-interference-plus-noise-ratio (SINR) at the output of the destination receiver. The waveforms are designed to occupy the whole available bandwidth of the device, therefore providing efficient utilization of the scarce UW-A spectrum resources. It is important to note that even in the absence of neighboring acoustic systems, the proposed cognitive waveform adaptation offers significant performance improvements in terms of receiver SINR and bit-error-rate (BER) due to the noise characteristics of the underwater acoustic channel.

The rest of the paper is organized as follows. In Section II we introduce the system model. Section III describes the receiver configuration for cognitive channelization and discusses the receiver developments. Section IV presents the proposed cognitive channelization algorithm for efficient spectrum management in underwater communications. Finally, Section $\mathrm{V}$ provides details on the experimental implementation of the proposed scheme and studies its performance in a real underwater environment (water test tank).

\section{SYSTEM MODEL}

We consider $K$ asynchronous multiplexed underwater signals that comprise binary antipodal information symbols $b_{k}(i) \in\{ \pm 1\}, k=1,2, \ldots K, i=0, \ldots, J-1$, at rate $1 / T$ modulated by digital waveforms $d_{k}(t)$ of length $L$ and duration $T, k=1,2, \ldots, K$. The $k$-th underwater signal can be written as

$$
x_{k}(t)=\sum_{i=0}^{J-1} b_{k}(i) \sqrt{E_{k}} d_{k}(t-i T) e^{j\left(2 \pi f_{c} t+\phi_{k}\right)},
$$

where $J$ is the data record size, $E_{k}$ is the transmitted energy per bit, $f_{c}$ is the carrier frequency, and $\phi_{k}, k=1,2, \ldots, K$, is the carrier phase relative to the $k$-th transmitter's local 
oscillator. Information symbols $b_{k}(i)$ are modulated by digital waveforms $d_{k}(t)=\sum_{l=0}^{L-1} s_{k}(l) g_{T_{d}}\left(t-l T_{d}\right), k=1,2, \ldots, K$ where $s_{k}(l) \in \frac{1}{\sqrt{L}}\{ \pm 1\}$ is the $l$-th code-bit of the digital waveform that uniquely identifies the $k$-th signal, $k=1,2, \ldots K$, and $g_{T_{d}}(\cdot)$ is a square-root raised cosine (SRRC) pulse of duration $T_{d}\left(T=L T_{d}\right)$. All signals are considered to propagate over independent time-varying multipath fading underwater acoustic channels with impulse response of the following general form

$$
h(\tau, t)=\sum_{n=0}^{N-1} a_{n}(t) \delta\left(\tau-\tau_{n}(t)\right),
$$

where $N$ denotes the total number of paths (assumed to be the same for all $K$ signals), $a_{n}(t)$ is the $n$-th path's amplitude and $\tau_{n}(t)$ is the time-varying path delay. The received passband signal can therefore be expressed as

$$
r(t)=\sum_{k=1}^{K} \sum_{n=0}^{N-1} a_{k, n}(t) x_{k}\left(t-\tilde{\tau}_{k, n}(t)\right)+n(t)
$$

where $\tilde{\tau}_{k, n}(t)$ denotes comprehensively the transmission delay and multipath propagation delay of the $n$-th path of the $k$ th signal, and $n(t)$ is additive noise. We assume negligible Doppler scaling. Therefore, path amplitudes $\alpha_{k, n}(t)$ and delays $\tilde{\tau}_{k, n}(t)$ can be modeled as time invariant during $J$ bit transmission periods.

If $\Delta f_{k}$ denotes the carrier frequency offset (CFO) between the receiver and the $k$-th transmitter, then after bandpass filtering and carrier demodulation the received baseband signal can be expressed as

$$
\begin{aligned}
r_{b}(t) & =r(t) e^{-j 2 \pi\left(f_{c}+\Delta f_{k}\right) t} \\
& =\sum_{k=1}^{K} \sum_{n=0}^{N-1} \tilde{a}_{k, n} \sum_{i=0}^{J-1} b_{k}(i) d_{k}\left(t-i T-\tilde{\tau}_{k, n}\right)+\tilde{n}(t)
\end{aligned}
$$

where $\tilde{a}_{k, n}=\sqrt{E_{k}} a_{k, n} e^{-j\left(2 \pi f_{c} \tilde{\tau}_{k, n}-\phi_{k}+2 \pi \Delta f_{k}\right)}$ and $\tilde{n}(t)$ is the baseband equivalent additive noise. In contrast to radio frequency communications, $\mathrm{CFO}$ in underwater can be considered negligible when compared to the carrier frequency. As a result, $\Delta f_{k}, k=1,2, \ldots, K$ can be treated as a time-invariant channel phase during the channel coherence time.

\section{RECEIVER CONFIGURATION}

In this section we design and implement an adaptive interference suppressive receiver that deals with the problems of frame detection, symbol time synchronization, channel estimation, and maximum SINR filtering of the received signal in the UW-A channel. The proposed receiver structure executes jointly, blind synchronization, channel estimation and demodulation in a combined fashion, thus managing computational efficiency and multiple access interference resistance. Frame detection and phase ambiguities due to blind channel estimates are resolved with limited pilot signaling. (Experimental studies showed that $10 \%$ of the information data is sufficient for pilot purposes.)

A training sequence of $P$ unmodulated symbols (e.g., the all-1 sequence) is used for detection of the transmission frame and coarse synchronization for the signal of interest. Specifically, let $f_{s}=\frac{1}{T_{s}}$ and $I=\frac{T_{d}}{T_{s}}$ be the receiver's sampling rate and the number of samples per waveform codebit used for interpolation at the transmitter, respectively. Then, for the $m$-th received sample $r_{b}[m] \triangleq r_{b}\left(m T_{s}\right)$, we calculate a moving average over a variable window of length $P_{\text {win }}$, as follows, $R[m]=\sum_{l=0}^{P_{\text {win }}-1} r_{b}[m+l] r_{b}^{*}[m+l+L I]$. Let $W[m] \triangleq \frac{|R[m]|}{P[m]}$ denote a coarse timing metric, where $P[m] \triangleq$ $\sum_{l=0}^{P_{\text {win }}-1}\left|r_{b}[m+l]\right|^{2}$ is used for normalization purposes. We detect the beginning of a frame if $W[m]>\Theta$ for a number of consecutive samples and a user-defined threshold $\Theta$. We note that $W[\mathrm{~m}]$ reaches a plateau that ideally (i.e., in the absence of intersymbol interference (ISI) and multiple access interference (MAI)) extends over $P \cdot L \cdot I$ samples.

Without loss of generality, the frame detection algorithm provides a timing uncertainty, $\tilde{\tau}_{k, n}$ which is a multiple of the basic SRRC pulse duration $T_{d}$. That is, let $\tilde{\tau}_{k, n}=\kappa_{k, n} T_{d}$ for some $\kappa_{k, n} \in\{0,1, \ldots, L-1\}$. We assume that the symbol duration is chosen to be greater than the multipath spread of the channel $T=L T_{d}>>T_{m}$, thus ISI is not destructive, and $N \leq(L / 2)+1$.

After pulse matched filtering and sampling at rate $1 / T_{d}$ of the received sampled signal, the discrete time received signal $r_{s}[m]$ over the multipath-extended period of $(L+N-1) T_{d}$ is given by

$$
\begin{aligned}
r_{s}[m] & =\sum_{i=m I}^{((m+1)+N-1) I} r_{b}[i] \\
& =\sum_{k=1}^{K} \sum_{n=0}^{N-1} \tilde{a}_{k, n} b_{k}\left(\left\lfloor\frac{m-\kappa_{k, n}}{L+N-1}\right\rfloor\right) \\
& \cdot s_{k}\left(\left\lfloor\left(m-\kappa_{k, n}\right) \%(L+N-1)\right\rfloor\right)+\tilde{n}[m]
\end{aligned}
$$

where $\tilde{n}[m]$ is baseband noise, $\lfloor\cdot\rfloor$ denotes the floor operator, and $\%(L+N-1)$ denotes the modulo- $(L+N-1)$ operation.

Next we address the problem of estimating the time delay $\tilde{\tau}_{1, n}$, and complex channel gain $\tilde{a}_{1, n}$ of the $n$-th multipath component, $n=0,1, \ldots, N-1$ of the signal of interest. Towards this end, we develop a linear RAKE-type receiver for combined synchronization, channel estimation, and demodulation of the information bits of the signal of interest, under the assumption of unknown code-bit timing, unknown energy, delay, and waveform code-bits of all other co-existing $(K-1)$ signals.

Effective demodulation is achieved if the received vector includes at least one complete information symbol. This is always the case, regardless of the delay of the desired transmission, if the received vector is at least $2 L$-long. In this paper, we follow the developments in [8] and build an effective receiver that employs lower-order filters of length- $(L+N-1)$, and avoids the computational complexity of longer filters. In particular, we propose an interference resistant synchronization scheme that is based on the principles of subspace channel estimation.

Assuming timing uncertainty in $[0, T)$ due to frame detection the $(L+N-1) \times 1$ input vector $\mathbf{r}_{\mathbf{i}} \triangleq$ $\left[r_{s}[i L], \ldots, r_{s}[(i+1) L+N-2]\right]^{T} \in \mathbb{C}^{L+N-1}$ can be written as follows

$$
\begin{aligned}
\mathbf{r}_{i} & =\left[b_{1}(i) \mathbf{v}_{1}^{\kappa_{1, n}}+b_{1}(i-1) \mathbf{v}_{1}^{\kappa_{1, n}-}+b_{1}(i+1) \mathbf{v}_{1}^{\kappa_{k, n}+}\right] \\
& +\sum_{k=2}^{K}\left[b_{k}(i) \mathbf{v}_{k}^{\kappa_{k, n}}+b_{k}(i-1) \mathbf{v}_{k}^{\kappa_{k, n}-}+b_{k}(i+1) \mathbf{v}_{k}^{\kappa_{k, n}^{+}}\right] \\
& +\tilde{\mathbf{n}}_{i}, \quad i=0,1, \ldots, J-1 .
\end{aligned}
$$

where $\tilde{\mathbf{n}}_{i} \triangleq[\tilde{n}[i L], \ldots, \tilde{n}[(i+1) L+N-2]]^{T}$ is modeled as zero-mean additive white Gaussian noise (AWGN) vector with 
autocorrelation matrix $\mathbf{R}_{N}=\sigma^{2} \mathbf{I}_{(L+N-1) \times(L+N-1)}$. In (6), $\mathbf{v}_{k}^{\kappa_{k, n}}$ is the space-time (ST) RAKE vector of the $k$-th signal that is defined as

$$
\mathbf{v}_{k}^{\kappa_{k, n}} \triangleq \sum_{n=0}^{N-1} \tilde{a}_{k, n}[\underbrace{[0, \ldots, 0]}_{n+\kappa_{k, n}},\left[s_{k}(0), \ldots, s_{k}(L-1)\right], \underbrace{[0, \ldots, 0]}_{N-\kappa_{k, n}-n-1}]_{(7)}^{T}
$$

where, we recall, $\kappa_{k, n}$ is modeled as a deterministic unknown that takes values in $\{0,1, \ldots, L-1\}$ and $\mathbf{v}_{k}^{\kappa_{k, n^{+}}}, \mathbf{v}_{k}^{\kappa_{k, n^{-}}}$are defined similarly to (7) i.e., $\mathbf{v}_{k}^{\kappa_{k, n}+} \triangleq$ $\sum_{n=0}^{N-1} \tilde{a}_{k, n}[\underbrace{[0, \ldots, 0]}_{n+\kappa_{k, n}},\left[s_{k}(0), \ldots, s_{k}\left(L-\kappa_{k, n}-1\right)\right], \underbrace{[0, \ldots, 0]}_{N-n-1}]^{T}$, and $\mathbf{v}_{k}^{\kappa_{k, n}-} \triangleq \sum_{n=0}^{N-1} \tilde{a}_{k, n}[\underbrace{[0, \ldots, 0]}_{n},\left[s_{k}\left(L-\kappa_{k, n}\right), \ldots, s_{k}(L-1)\right]$, $\underbrace{[0, \ldots, 0]}_{N-\kappa_{k, n}-n-1}]^{T}, k=1, \ldots, K, \quad n=0, \ldots, N-1$.

For simplicity in notation, we assume, for a moment, that $\kappa_{1, n}=0$ (i.e., the timing ambiguity $\kappa_{1, n}$ of the signal of interest is known and compensated). Then, we can rewrite $\mathbf{r}_{i}$ as follows

$$
\mathbf{r}_{i}=b_{1}(i) \mathbf{S}_{1} \mathbf{h}_{1}+\mathbf{M A I} \mathbf{I}_{i}+\mathbf{n}_{i}, \quad i=0,1, \ldots, J-1
$$

where $\mathbf{M A I}_{i}$ accounts for the space-time MAI, $\mathbf{h}_{1}=$ $\left[\tilde{a}_{1,0}, \tilde{a}_{1,1}, \ldots, \tilde{a}_{1, N-1}\right]^{T}$ is the unknown channel coefficient vector, and $\mathbf{S}_{1} \triangleq\left[\begin{array}{ccc}\mathbf{s}_{1} & \ldots & \mathbf{0} \\ \vdots & \ddots & \vdots \\ \mathbf{0} & \ldots & \mathbf{s}_{1}\end{array}\right]$ is the $(L+N-1) \times N$ waveform matrix.

We incorporate the timing uncertainty $\kappa_{1, n}$ in the synchronization-channel estimation process as follows. We quantize the $[0, T)$ interval into a finite number of $L$ subintervals/hypotheses and form $L$ received vectors of length $(L+N-1)$, each corresponding to a delay subinterval. If $\mathbf{r}_{i}^{(l)}$ denotes the received vector over the $i$-th information symbol period, that is observed with a delay of $l$ waveform code-bits (l-th subinterval), then

$$
\begin{aligned}
\mathbf{r}_{i}^{(l)} \triangleq & {\left[r_{s}[i L+l], r_{s}[i L+l+1], \ldots,\right.} \\
& \left.r_{s}[(i+1) L+N+l-2]\right]^{T} \\
& l=0,1, \ldots, L-1, \quad i=0,1, \ldots J-1 .
\end{aligned}
$$

For any given $l, l=0, \ldots, L-1$ we construct $\mathbf{R}^{(l)} \triangleq$ $E\left\{\mathbf{r}_{i}^{(l)} \mathbf{r}_{i}^{(l)^{H}}\right\}$ i.e., the autocorrelation matrix of the received vector $\mathbf{r}_{i}^{(l)}$. By applying eigenvalue decomposition on $\mathbf{R}^{(\mathbf{l})}$ we obtain $\mathbf{R}^{(l)}=\mathbf{Q}^{(l)} \mathbf{\Lambda}^{(l)} \mathbf{Q}^{(l)}$ where the columns of $\mathbf{Q}^{(l)}$ are the eigenvectors of $\mathbf{R}^{(l)}$ and $\boldsymbol{\Lambda}^{(l)}$ is a diagonal matrix containing the eigenvalues of $\mathbf{R}^{(l)}$. We use the eigenvectors that correspond to the $(L+N-1)-(2 K-1)$ smallest eigenvalues, we construct the matrix $\mathbf{U}^{(l)} \in \mathbb{C}^{(L+N-1) \times(L+N-1)-(2 K-1)}$, and we select as a channel estimate-candidate the vector $\mathbf{h}_{1}^{(l)}$ that minimizes the projection $\left(\mathbf{S}_{1} \mathbf{h}_{1}^{(l)}\right)^{H} \mathbf{U}^{(l)}=\mathbf{0}$ subject to $\left\|\mathbf{h}_{1}^{(l)}\right\|=1$ i.e., the vector that corresponds to the smallest eigenvalue of matrix $\mathbf{M}^{(l)}=\mathbf{S}_{1}^{H} \mathbf{U}^{(l)} \mathbf{U}^{(l)^{H}} \mathbf{S}_{1}$, that we denote as $\lambda_{m i n}^{(l)}$. We repeat this process for $l=0, \ldots, L-1$ and create $L$ channel candidates. Among those candidates we choose as our channel estimate the one that corresponds to the smallest eigenvalue among $\left\{\lambda_{\min }^{(0)}, \ldots, \lambda_{\min }^{(L-1)}\right\}$.

A byproduct of the above subspace channel estimation technique is the evaluation of $\mathbf{R}^{(l)}$ for $l=0, \ldots, L-1$ which are needed for the combined synchronization and demodulation scheme that follows. The basis behind the proposed combined synchronization/demodulation RAKE-type scheme is that maximum-SINR filtering design criteria are highly dependent on synchronization, under the assumption of channel availability. Here, we consider a combination of the RAKE principles with a linearly constrained minimum variance filter [9] $\mathbf{w}$ that minimizes the filter's output energy and at the same time remains distortionless to some particular vector direction $\mathbf{d}$ (i.e., $\mathbf{w}^{H} \mathbf{d}=1$ ). In this context, under the assumption of a perfectly synchronized system $\left(\kappa_{1, n}=0\right)$, $\mathbf{v}_{|| 1||} \triangleq \mathbf{v}_{1}^{0} /\left\|\mathbf{v}_{1}^{0}\right\|$ with $\mathbf{v}_{1}^{0}=\mathbf{S}_{1} \mathbf{h}_{1}$ is the desired direction of interest. If $\mathbf{R} \triangleq E\left\{\mathbf{r}_{i} \mathbf{r}_{i}^{H}\right\}$ is the autocorrelation matrix of the $i$-th received vector $\mathbf{r}_{i}$, then the maximum-SINR RAKE-type MVDR filter is given by $\mathbf{w}_{\mathbf{v}_{|| 1||}, \mathrm{MVDR}}=\frac{\mathbf{R}^{-1} \mathbf{v}_{|| 1||}}{\mathbf{v}_{\| 1||}^{H} \mathbf{R}^{-1} \mathbf{v}_{|| 1||}}$.

However, in non-synchronized systems $\left(\kappa_{1, n} \neq 0\right)$ the actual vector direction of the desired signal is $\mathbf{v}_{1}^{\kappa_{1, n}}$ for some unknown $\kappa_{1, n}$. Therefore, the lower the cross-correlation between $\mathbf{v}_{1}^{0}$ and the actual $\mathbf{v}_{1}^{\kappa_{1, n}}$, the lower the output energy of the RAKE-type MVDR filter will be, since a significant portion of the desired signal will be suppressed. On the other hand, high cross-correlation between $\mathbf{v}_{1}^{0}$ and $\mathbf{v}_{1}^{\kappa_{1, n}}$ will result in maximum output energy of the receiver filter. Both observations help us develop a receiver structure that achieves combined synchronization/demodulation through the following proposition.

Proposition 1: Provided that we consider power-controlled systems that employ a RAKE-type MVDR receiver and if the SINRs of the received signals are sufficiently large then

$$
\mathbf{w}_{\mathbf{v}_{\||1|, \mathrm{MVDR}}^{(l)^{H}}} \mathbf{R}^{(l)} \mathbf{w}_{\mathbf{v}_{\|1\|, \mathrm{MVDR}}^{(l)}}^{(l)}, \quad l=0,1, \ldots L-1
$$

is maximized for $l=\kappa_{1, n}$, where $\mathbf{R}^{(l)}$ is the input autocorrelation matrix of the received vectors of the $l$-th sequence. In (9), $\mathbf{w}_{\mathbf{v}|| 1 \mid, \text { MVDR }}^{(l)}$ is a RAKE-type MVDR filter distortionless in $\mathbf{V}_{\|1\|}$ and evaluated based on the statistics of the input sequence $\left\{\mathbf{r}_{i}^{(l)}\right\}$, (i.e., $\mathbf{w}_{\mathbf{v}_{|| 1||}, \operatorname{MVDR}}^{(l)}=\mathbf{R}^{(l)^{-1}} \mathbf{v}_{\| 1||} / \mathbf{v}_{\|1\|}^{H} \mathbf{R}^{(l)^{-1}} \mathbf{v}_{\|1\|}$ ). In addition, effective demodulation of the desired signal of interest is achieved when the linear receiver operates on the synchronized input vector sequence $\left\{\mathbf{r}_{i}^{\left(\kappa_{1, n}\right)}\right\}$.

In practice, autocorrelation matrix $\mathbf{R}^{(l)}, l=0,1, \ldots, L-1$ is sample-average estimated, $\hat{\mathbf{R}}^{(l)}=\frac{1}{J} \sum_{i=0}^{J-1} \mathbf{r}_{i}^{(l)} \mathbf{r}_{i}^{(l)^{H}}$, where $J$ denotes the available data record. In underwater communications due to the rapidly changing nature of the environment we have to deal with small data records that satisfy the channel's coherence time $(\sim 1 \mathrm{~s})$. In our experiments data record $J$ is chosen as $J>(L+N-1)$. To ensure full rank of $\mathbf{R}^{(l)}$ we use a diagonally loaded version of the sample average estimate of the autocorrelation matrix (i.e., $\hat{\mathbf{R}}^{(l)}=1 / J \sum_{i=0}^{J-1} \mathbf{r}_{i}^{(l)} \mathbf{r}_{i}^{(l)}{ }^{H}+\gamma \mathbf{I}_{L+N-1}$, where $\gamma$ is the diagonal loading factor to be appropriately (heuristically) chosen [10]).

Before proceeding with recovery of the transmitted information bits $b_{1}(i)$ of the signal of interest, we need to resolve 
the phase ambiguity introduced in $\mathbf{v}_{1}^{0}$. The adopted blind subspace channel estimation technique results in phase ambiguous $\mathbf{v}_{|| 1||}$, thus the overall receiver filter $\mathbf{w}_{\mathbf{v}_{\||1|, M V D R}}^{\left(\kappa_{1, n}\right)}$ is also phase ambiguous. For this purpose we will take advantage of the length- $P$ pilot information bit sequence we used for frame detection. The mean-square (MS) optimum phase estimate that minimizes the mean-square error (MSE) between the spacetime RAKE-type MVDR filter and the desired information bit $b_{1}$ (i.e., $\left.\sum_{i=0}^{P-1}\left|\left(\mathbf{w}_{\mathbf{v}_{|| 1 \mid 1, \mathrm{MVDR}}}^{\left(\kappa_{1, n}\right)} e^{j \phi}\right)^{H} \mathbf{r}_{i}^{\left(\kappa_{1, n}\right)}-b_{1}(i)\right|^{2}\right)$ is given by $[11]$

$$
\hat{\phi}=\operatorname{angle}\left\{\mathbf{w}_{\mathbf{v}_{\|1\|, \mathrm{MVDR}}}^{\left(\kappa_{1, \mathrm{n}}\right)^{\mathrm{H}}} \sum_{\mathrm{i}=0}^{\mathrm{P}-1} \mathbf{r}_{\mathrm{i}}^{\left(\kappa_{1, \mathrm{n}}\right)} \mathrm{b}_{1}^{*}(\mathrm{i})\right\} .
$$

After phase compensation, bit decisions are made over the filtered data as follows

$$
\hat{b}_{1}(i)=\operatorname{sgn}\left(\Re\left\{\left(\mathbf{w}_{\mathbf{v}_{\|1\|, \mathrm{MVDR}}}^{\left(\kappa_{1, \mathrm{n}}\right)} \mathrm{e}^{\mathrm{j} \hat{\phi}}\right)^{\mathrm{H}} \mathbf{r}_{\mathrm{i}}^{\left(\kappa_{1, \mathrm{n}}\right)}\right\}\right)
$$

for $i=0,1, \ldots, J-1$, and $\operatorname{sgn}\{\cdot\}$ denotes the sign operation.

\section{OPTIMAL CHANNELIZATION}

The pre-detection SINR attained at the RAKE-type MVDR receiver filter output $\left(\mathbf{w}_{\operatorname{maxSINR}}=\mathbf{w}_{\mathbf{v}_{\|\mid 1\|, \mathrm{MVDR}}}^{\left(\kappa_{1, n}\right)^{H}} e^{j \hat{\phi}}\right)$ can be calculated as

$$
\begin{aligned}
\mathrm{SINR} & \triangleq \frac{E\left\{\left|\mathbf{w}_{\operatorname{maxSINR}}^{H}\left(b_{1}(i) \mathbf{H}_{1} \mathbf{s}_{1}\right)\right|^{2}\right\}}{E\left\{\left|\mathbf{w}_{\operatorname{maxSINR}}^{H}\left(\mathbf{M A I} \mathbf{I}_{i}+\mathbf{n}_{i}\right)\right|^{2}\right\}} \\
& =\mathbf{s}_{1}^{H} \mathbf{H}_{1}^{H} \mathbf{R}_{I+N}^{-1} \mathbf{H}_{1} \mathbf{s}_{1}=\mathbf{s}_{1}^{H} \mathbf{Q}_{1} \mathbf{s}_{1}
\end{aligned}
$$

where $\mathbf{Q}_{1}=\mathbf{H}_{1}^{H} \mathbf{R}_{I+N}^{-1} \mathbf{H}_{1}, \mathbf{Q}_{1} \succ 0$, and $\mathbf{H}_{1} \in \mathbb{C}^{(L+N-1) \times L}$ is the multipath fading channel matrix of the signal of interest that is given by $\mathbf{H}_{1}=\sum_{n=0}^{N-1} \tilde{a}_{1, n}\left[\begin{array}{l}\mathbf{0}_{n \times L} \\ \mathbf{I}_{L \times L} \\ \mathbf{0}_{(N-n-1) \times L}\end{array}\right]$. In practice, we utilize estimates of $\mathbf{R}_{I+N}$ based on a finite data record size. In particular, $\mathbf{R}_{I+N}$ is sample-average estimated over $D>J$ signal-of-interest-absent snapshots of the received signal i.e., $\hat{\mathbf{R}}_{I+N}=\frac{1}{D} \sum_{i=0}^{D-1}\left(\mathbf{M A I} \mathbf{I}_{i}+\mathbf{n}_{i}\right)\left(\mathbf{M A I} \mathbf{I}_{i}+\mathbf{n}_{i}\right)^{H}$. The main objective is to design and use the best channel waveform $\mathbf{s}_{1}$ i.e., the one that maximizes $\operatorname{SINR}=\mathbf{s}_{1}^{\mathrm{H}} \mathbf{Q}_{1} \mathbf{s}_{1}$, therefore achieving cognitive channelization. In other words, we are looking for the best channel waveform $\mathbf{s}_{1}^{o p t}$ that solves the following optimization problem

$$
\begin{array}{ll}
\underset{\mathbf{s}_{1} \in \mathbb{R}^{L}}{\operatorname{maximize}} & \mathbf{s}_{1}^{H} \mathbf{Q}_{1} \mathbf{s}_{1} \\
\text { subject to } & \mathbf{s}_{1}^{H} \mathbf{s}_{1}=1 .
\end{array}
$$
tion.

The solution to (13) is provided by the following proposi-

Proposition 2: Let $\mathbf{q}_{1}, \mathbf{q}_{2}, \ldots, \mathbf{q}_{L}$ be the eigenvectors of $\mathbf{Q}_{1}$ with corresponding eigenvalues $\lambda_{1} \geq \lambda_{2} \geq \cdots \geq \lambda_{L}$. The normalized waveform that maximizes (13) is the eigenvector $\mathbf{s}_{1}^{o p t}=\mathbf{q}_{1}$ that corresponds to the maximum eigenvalue $\lambda_{1}$ of $\mathbf{Q}_{1}$.

\section{EXPERIMENTAL SETUP AND RESUlts}

Underwater cognitive channelization is evaluated with inhouse built software-defined acoustic (SDA) modems [12] in an experimental testbed setup of $K=2$ users/signals. Experiments are conducted in a water test tank of dimensions $8 \mathrm{ft} \times 2.5 \mathrm{ft} \times 2 \mathrm{ft}$ as depicted in Fig. 2 .

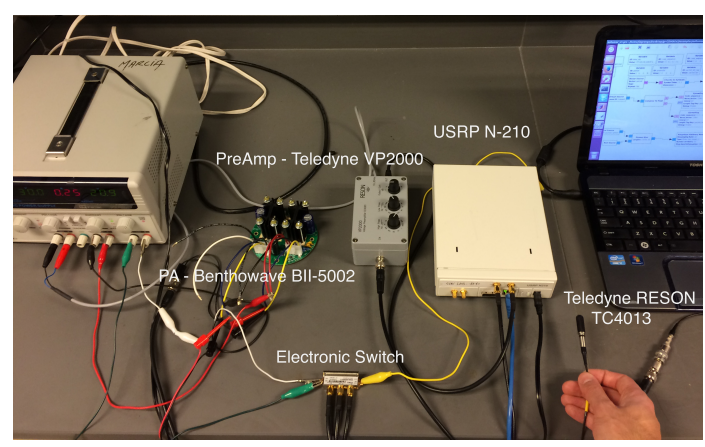

Fig. 1. In-house built USRP-based software-defined acoustic modem.

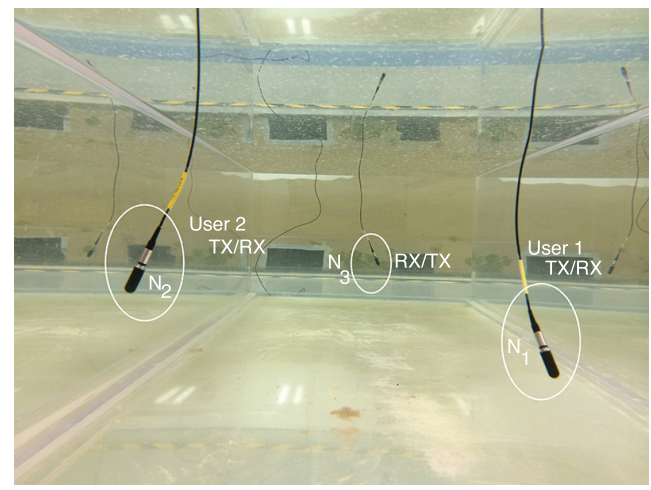

Fig. 2. Testbed deployment of $K=2$ users in a water test tank.

Figure 1 illustrates the prototype structure of the softwaredefined acoustic modem. The SDA modem is based on a USRP - N210 platform that is equipped with both LFTX and LFRX daughtercards and operates as a half-duplex transceiver in the frequency range from $\mathrm{DC}$ to $30 \mathrm{MHz}$. The amplifying output power of LFTX is enhanced by the addition of a linear wideband power amplifier (PA), Benthowave BII - 5002, that has maximum output power of $192 \mathrm{~dB}$, and can support up to $300 \mathrm{kHz}$ bandwidth. At the receiver chain of the modem, we use the voltage preamplifier (PreAmp), Teledyne VP2000, to provide low-noise performance in the desired frequency range with a range of bandpass filters, and adjustable gain selection. Half-duplex operation is achieved through an electronic switch (Mini-Circuits ZX80 - DR230+) that is controlled by a General Purpose Input/Output (GPIO) digital pin available on the USRP's daughterboards. Finally, the electronic switch is connected to an acoustic transducer (Teledyne RESON TC4013) that offers an operational frequency range from $1 \mathrm{~Hz}$ to $170 \mathrm{kHz}$.

The USRP-based acoustic modem is connected to a hostPC with Gigabit Ethernet (GigE) and is controlled by a host driver, known as UHD (Universal Hardware Driver). At the PC side, we select GNU Radio as the software platform for baseband processing. GNU Radio is a software framework that offers libraries of primitive building $\mathrm{C}++$ blocks for implementing basic wireless communication functionalities. We exploit the available libraries and implement both transmitter and receiver functionalities for underwater cognitive channelization in the GNU Radio environment with custom out-of-tree $\mathrm{C}++$ blocks. We then wrap these signal processing blocks into a "flowgraph" with the aid of a Python interface.

The testbed consists of three SDA modems that are submerged in a water test tank (Fig. 2). Two of the nodes, $N_{1}$, and $N_{2}$ act as transmitters, and $N_{3}$ as a receiver. A feedback link 


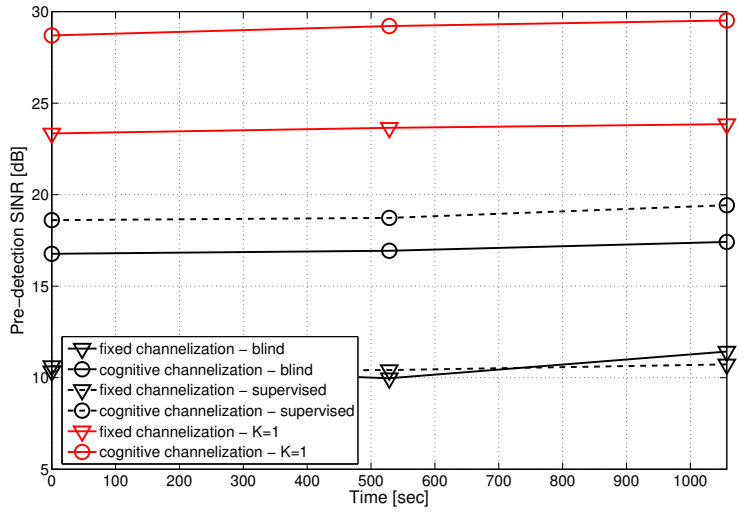

Fig. 3. Cognitive channelization is compared with fixed channelization in terms of pre-detection SINR. The depicted SINR gains consider three different setups: (i) the proposed blind receiver configuration (ii) a fully supervised receiver and (iii) a single user $K=1$ case.

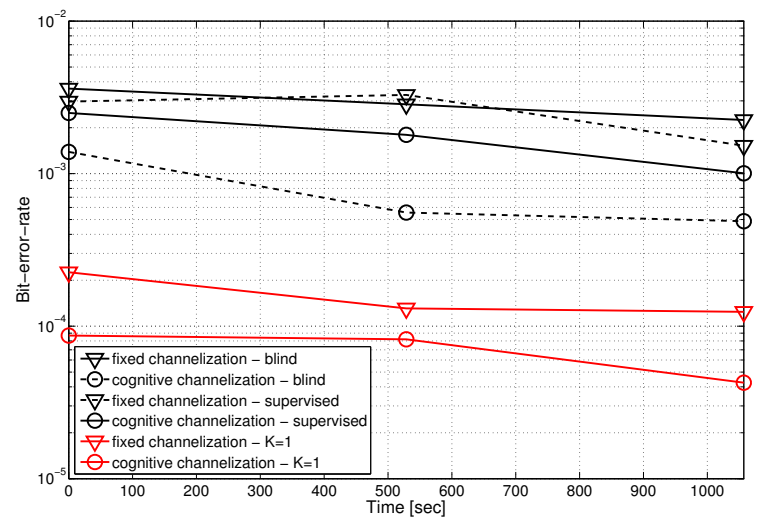

Fig. 4. BER measured at the receiver for the cases of cognitive and fixed channelization. Performance gains of cognitive channelization are reported for three different setups: (i) the proposed blind receiver configuration (ii) a fully supervised receiver and (iii) a single user $K=1$ case.

for exchanging the optimal channel waveform is established between $N_{1}$ and $N_{3}$. All three nodes operate on the same frequency band $91-99 \mathrm{kHz}$ and use random binary waveforms of length $L=63$. Both transmitters use SRRC pulses of duration $T_{d}=0.16384 \mathrm{~ms}$, and roll-off factor $\alpha=0.35$. We set the number of channel paths $N=20$ and measured a mutlipath spread of 31 waveform bits or $5.1 \mathrm{~ms}$. Both $N_{1}$ and $N_{2}$ use the same transmission power and are positioned $5.5 \mathrm{ft}$ apart from $N_{3}$. The distance between $N_{1}$ and $N_{2}$ is set at $1.5 \mathrm{ft}$.

In the following, we present BER and SINR experimental results from three different setups. First, we consider a single transmitter $N_{1}$ that operates in the absence of $N_{2}$. Second, we investigate the case of $K=2$ users. Nodes $N_{1}$ and $N_{2}$ transmit simultaneously, at the same frequency band, while $N_{3}$ uses a control channel at a different frequency band to periodically communicate to $N_{1}$ a new channel waveform. For both cases we consider a packet size of $J=144$ bits, and $P=16$ pilot bits. Finally, we compare the proposed blind receiver with a supervised receiver structure that uses a zero-padded, 511-length PN-sequence based preamble (of total duration $104 \mathrm{~ms}$ ) to achieve frame-detection, synchronization, and channel estimation.

Figure 3 illustrates SINR experimental results for the three setups. For each setup we consider two different scenarios called cognitive and fixed channelization, respectively. Cognitive channelization assumes that the transmitter node $\mathrm{N}_{1}$ adapts his channel waveform $\mathbf{s}_{1}$ into $\mathbf{s}_{1}^{\mathrm{opt}}$, based on the interference profile of $\mathrm{N}_{2}$ and the additive noise. In the absence of $N_{2}$, waveform adaptation is based only on the noise profile. For the fixed channelization scenario, $N_{1}$ does not change the pre-set waveform $\mathbf{s}_{1}$. We observe that for all three different setups, cognitive channelization significantly outperforms fixed channelization in terms of receiver pre-detection SINR.

Figure 4 presents the corresponding BER experimental results for cognitive and fixed channelization and demonstrates the performance gains of the first method for both single and $K=2$-users setups. In the $K=2$-users setup we choose to assign node $N_{2}$ to an initial waveform that exhibits high correlation $(60 \%)$ with the waveform assigned to $N_{1}$, emulating this way a high-interference environment.

Our experimental studies in a real underwater environment showed that (i) underwater cognitive channelization is always beneficial regardless of the supervised or unsupervised (blind) nature of the receiver; (ii) even in the absence of interference from other signals/users, cognitive channelization improves receiver performance due to the fact that it efficiently adapts to channel conditions; (iii) the proposed blind receiver scheme performs very close to its fully supervised counterpart while at the same time offers significantly better $(50 \%)$ bandwidth utilization.

\section{REFERENCES}

[1] T. Melodia, H. Kulhandjian, L. Kuo, and E. Demirors, "Advances in Underwater Acoustic Networking," in Mobile Ad Hoc Networking: Cutting Edge Directions, 2nd ed. Inc., Hoboken, NJ: John Wiley and Sons, 2013, pp. 804-852.

[2] Y. Luo, L. Pu, M. Zuba, Z. Peng, and J. Cui, "Challenges and opportunities of underwater cognitive acoustic networks," IEEE Transactions on Emerging Topics in Computing, vol. PP, no. 99, pp. 1-1, 2014.

[3] K. Gao, S. N. Batalama, D. A. Pados, and J. D. Matyjas, "Cognitive code-division channelization," IEEE Trans. on Wireless Commun., vol. 10, pp. 1090-1097, 2011.

[4] L. Ding, K. Gao, T. Melodia, S. N. Batalama, D. A. Pados, and J. D. Matyjas, "All-spectrum cognitive networking through joint distributed channelization and routing," IEEE Trans. on Wireless Commun., vol. 11, pp. 5394-5405, 2013.

[5] H. P. Tan, W. K. Seah, and L. Doyle, "Exploring cognitive techniques for bandwidth management in integrated underwater acoustic systems," in Proc. of MTS/IEEE OCEANS, April 2008.

[6] N. Baldo, P. Casari, and M. Zorzi, "Cognitive spectrum access for underwater acoustic communications," in Proc. of IEEE ICC, May 2008.

[7] D. Torres, Z. Charbiwala, J. Friedman, and M. Srivastava, "Spectrum signaling for cognitive underwater acoustic channel allocation," in Proc. of IEEE INFOCOM, March 2010.

[8] I. N. Psaromiligkos, S. N. Batalama, and M. J. Medley, "Rapid combined synchronization/demodulation structures for ds-cdma systems - part i: Algorithmic developments," IEEE Trans. on Commun., vol. 51, pp. 983-994, 2003.

[9] S. Haykin, "Adaptive filter theory." Prentice-Hall, NJ: Englewood Cliffs, 1991.

[10] B. D. Carlson, "Covariance matrix estimation errors and diagonal loading in adaptive arrays," IEEE Trans. Aerosp. Electron. Syst., vol. 24, pp. 397-401, 1988.

[11] G. Karystinos and D. A. Pados, "Supervised phase correction of blind space-time ds-cdma channel estimates," IEEE Trans. on Commun., vol. 55, pp. 1009-1020, 2007.

[12] E. Demirors, G. Sklivanitis, G. E. Santagati, T. Melodia, and S. N. Batalama, "Design of a software-defined underwater acoustic modem with real-time physical layer adaptation capabilities," in Proc. of ACM Intl. Conf. on Underwater Networks \& Systems (WUWNet), November 2014. 